\title{
A HARPSS Polysilicon Vibrating Ring Gyroscope
}

\author{
Farrokh Ayazi, Member, IEEE, and Khalil Najafi, Fellow, IEEE
}

\begin{abstract}
This paper presents the design, fabrication, and testing of an 80- $\mu \mathrm{m}$-thick, $1.1 \mathrm{~mm}$ in diameter high aspect-ratio (20:1) polysilicon ring gyroscope (PRG). The vibrating ring gyroscope was fabricated through the high aspect-ratio combined poly and single-crystal silicon MEMS technology (HARPSS). This all-silicon single-wafer technology is capable of producing electrically isolated vertical electrodes as tall as the main body structure (50 to 100's ( $\mu \mathrm{m}$ tall) with various size air-gaps ranging from submicron to tens of microns. A detailed analysis has been performed to determine the overall sensitivity of the vibrating ring gyroscope and identify its scaling limits. An open-loop sensitivity of $200 \mu \mathrm{V} / \mathrm{deg} / \mathrm{s}$ in a dynamic range of $\pm 250 \mathrm{deg} / \mathrm{s}$ was measured under low vacuum level for a prototype device tested in hybrid format. The resolution for a PRG with a quality factor $(Q)$ of 1200, drive amplitude of $0.15 \mu \mathrm{m}$, and sense node parasitic capacitances of $2 \mathrm{pF}$ was measured to be less than $1 \mathrm{deg} / \mathrm{s}$ in $1 \mathrm{~Hz}$ bandwidth, limited by the noise from the circuitry. Elimination of the parasitic capacitances and improvement in the quality factor of the ring structure are expected to reduce the resolution to 0.01 $\operatorname{deg} / \mathrm{s} /(\mathbf{H z})^{0.5}$.

[627]
\end{abstract}

Index Terms-Deep trench etching, gyroscope, high aspect-ratio, microelectromechanical systems (MEMS), silicon micromachining, vibratory gyroscope, yaw rate sensor.

\section{INTRODUCTION}

$\mathbf{S}$ ILICON-MICROMACHINED vibratory gyroscopes have received a lot of attention during the past few years as many applications exist for these miniature devices. They can be used either as a low-cost companion with micromachined accelerometers to provide heading information for inertial navigation purposes or stand alone and be used in other applications where rotation rate needs to be measured; examples of these being automotive applications such as traction control systems, ride stabilization and roll-over detection; some consumer electronic applications such as stabilization of pictures in digital video camera and inertial mouse in computers; robotics applications; and, a wide range of military applications such as guidance of missiles and platform stabilization. Much of the effort in developing micromachined gyroscopes has concentrated on "rate grade" devices for automotive applications, which require a rotation rate resolution and bias stability of about $0.5 \mathrm{deg} / \mathrm{s}$. However, several applications require improved

Manuscript received September 29, 2000; revised January 12, 2001. This work was supported by the Defense Advanced Research Projects Agency (DARPA) under Contracts DABT63-C-0111 and F30602-98-2-0231. Subject Editor O. Tabata.

F. Ayazi was with the Center for Integrated MicroSystems, Department of Electrical Engineering and Computer Science, University of Michigan, Ann Arbor, MI 48109-2122 USA. He is now with the School of Electrical and Computer Engineering, Georgia Institute of Technology, Atlanta, GA 30332-0250 USA.

K. Najafi is with the Center for Integrated MicroSystems, Department of Electrical Engineering and Computer Science, University of Michigan, Ann Arbor, MI 48109-2122 USA.

Publisher Item Identifier S 1057-7157(01)03947-6. performance, including inertial navigation and guidance of missiles. Achieving "tactical and inertial grade" performance levels [1], i.e., resolution and bias stability better than 1-10 $\mathrm{deg} / \mathrm{h}$ and high scale factor accuracy in a bandwidth of 10-100 $\mathrm{Hz}$, has proven to be a tough challenge and new technologies and approaches are being developed to allow micromachined gyroscopes achieve this level of performance. The clover-leaf gyroscope of the Jet Propulsion Laboratory (JPL) is an example of a precision bulk micromachined MEMS vibratory gyroscope with a reported resolution and bias stability of $\sim 10 \mathrm{deg} / \mathrm{h} \mathrm{[2]}$. Other examples of high performance silicon-micromachined vibratory gyroscopes are the silicon-on-glass tuning-fork gyroscope reported by the Charles Stark Draper Laboratory [3], the polysilicon surface micromachined gyroscope reported by the Institute of Micromachining and Information Technology (IMIT) in Germany [4], and Samsung's silicon-on-insulator (SOI) lateral gyroscope [5]. A comprehensive review of micromachined gyroscopes is given by the authors in [1].

This paper reports the design, fabrication, and testing of a high aspect-ratio (20:1) $80 \mu \mathrm{m}$ tall vibrating polysilicon ring gyroscope (PRG) [6], [7] fabricated using the high aspect-ratio combined poly and single-crystal silicon (HARPSS) MEMS technology [8]. HARPSS is a mixed-mode microfabrication technology that combines the best features of bulk micromachining with surface micromachining to produce hundreds of microns thick silicon structures with various size airgaps ranging from submicron to tens of microns. A detailed analysis has been performed to determine the overall sensitivity and noise floor of the vibrating ring gyroscope and identify its scaling limits. Several aspects of this analysis apply to other gyroscope sensing structures and are discussed in detail. It is shown through this analysis that the HARPSS technology provides features that are required to achieve high performance. An $80-\mu \mathrm{m}$ tall, $1.1 \mathrm{~mm}$ in diameter vibratory PRG has been successfully fabricated using this technology and test results obtained from the open-loop operation of a prototype gyroscope under vacuum are presented.

\section{The Vibrating Ring GyroscoPe}

The vibrating ring gyroscope, shown in Fig. 1, consists of a ring, eight semicircular support springs, and drive, sense and control electrodes [9]. Symmetry considerations require at least eight springs to result in a balanced device with two identical elliptically-shaped flexural modes that have equal natural frequencies and are $45^{\circ}$ apart from each other [10]. As shown in Fig. 2, the antinodes of the second flexural mode are located at the nodes of the first flexural mode. The ring is electrostatically vibrated into the primary flexural mode with a fixed amplitude. When device is subjected to rotation around its normal 


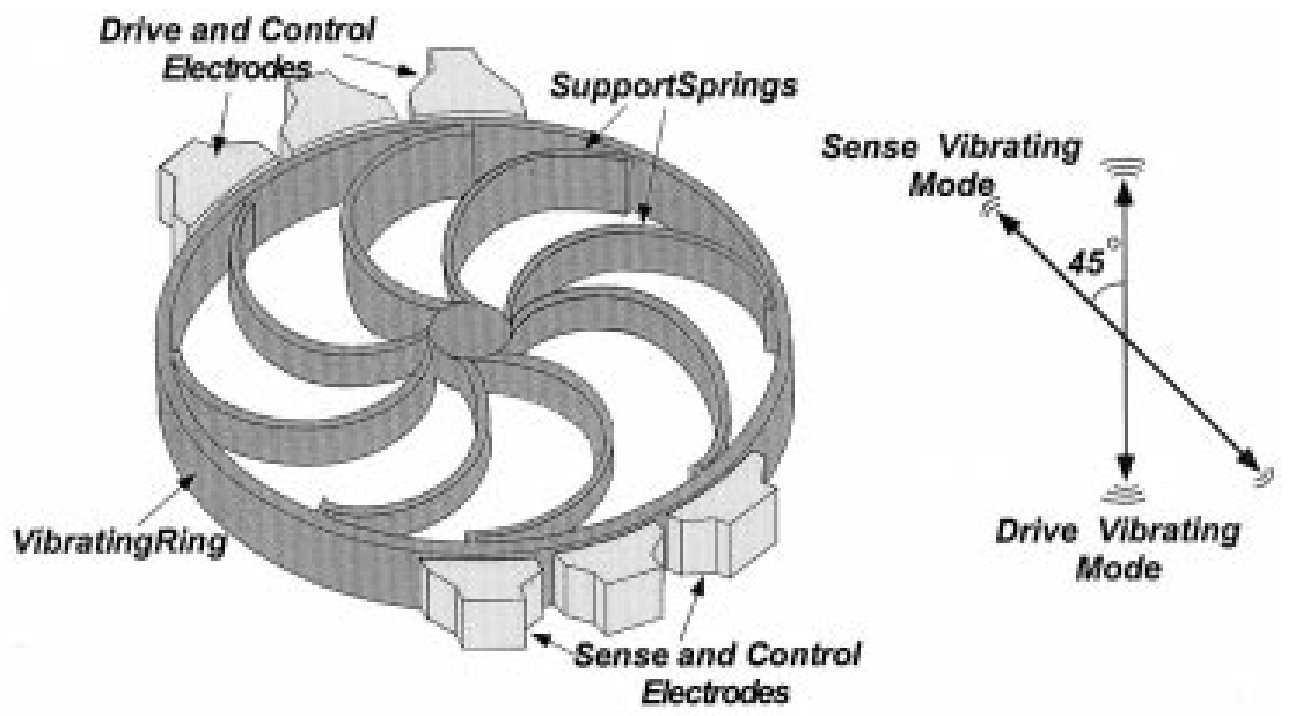

Fig. 1. Structure of a Vibrating Ring Gyroscope.

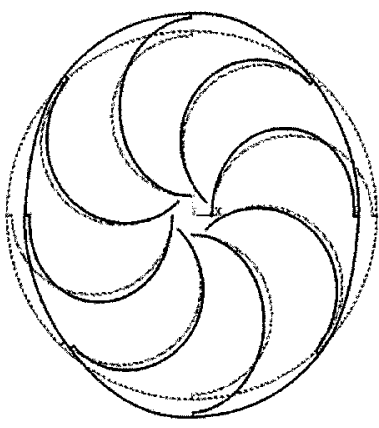

(a)

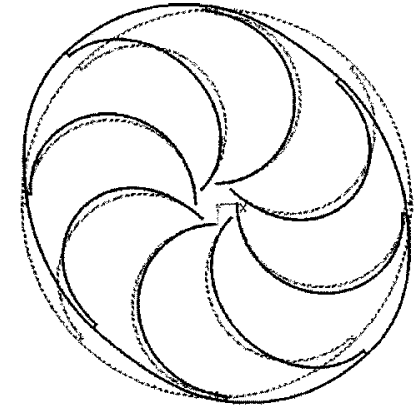

(b)
Fig. 2. Flexural modes of vibration used for operation of the vibrating ring gyroscope. (a) The first flexural mode and (b) the second flexural mode, located $45^{\circ}$ apart from the first mode with identical frequencies.

axis, Coriolis force causes energy to be transferred from the primary mode to the secondary flexural mode, which is located $45^{\circ}$ apart from the primary mode, causing amplitude to build up proportionally in the latter mode; this build-up is capacitively monitored. It has been shown in [10] that in the open-loop mode of operation, the amplitude of the second mode, or the sense mode ( $\left.q_{\text {sense }}\right)$, is proportional to the rotation rate and is given by

$$
q_{\text {sense }}=4 A_{g} \cdot \frac{Q}{\omega_{0}} \cdot q_{\text {drive }} \cdot \Omega_{z}
$$

where

$$
\begin{array}{ll}
A_{g} \approx 0.37 & \begin{array}{l}
\text { angular gain of the ring structure (which depends } \\
\text { on the geometry of the sensor and is very stable } \\
\text { over temperature and lifetime of the device); } \\
\text { quality factor of the mechanical structure; }
\end{array} \\
Q & \begin{array}{l}
\text { angular flexural resonance frequency; } \\
\omega_{0}
\end{array} \\
q_{\text {drive }} & \text { vibration amplitude of the drive mode; } \\
\Omega_{z} & \text { rotation rate. }
\end{array}
$$

The vibrating ring structure has some important features compared to other types of vibratory gyroscopes. First, it has a balanced symmetrical structure that is less sensitive to spurious vibrations. Only when the ring has mass or stiffness asymmetries can environmental vibrations induce a spurious response. Second, since two identical flexural modes of the structure "with nominally equal resonant frequencies" are used to sense rotation, the sensitivity of the sensor is amplified by the quality factor of the structure, resulting in higher sensitivity. Third, the vibrating ring is less temperature sensitive since the two flexural vibration modes are affected equally by temperature. Finally, electronic tuning of the structure is possible. Any frequency mismatch between the drive and sense resonance modes that occurs during fabrication process (due to mass or stiffness asymmetries) can be electronically compensated by use of the tuning electrodes that are located around the structure.

The first micromachined version of the vibrating ring gyroscope was fabricated by electroforming nickel into a thick polyimide (or photoresist) mold on a silicon substrate in a postcircuit process [9]. The gyroscope demonstrated a resolution of $\sim 0.5 \mathrm{deg} / \mathrm{s}$ in a $10-\mathrm{Hz}$ bandwidth limited by the readout electronic noise. The sensor was integrated with a low-input capacitance source-follower buffer and the amplifier on a silicon chip. The zero bias drift was smaller than $10 \%$ s over the temperature range $-40^{\circ} \mathrm{C}$ to $85^{\circ} \mathrm{C}$, and the sensitivity of the device varied by less than $3 \%$ over the same temperature range. Scale factor nonlinearity in a $\pm 100 \mathrm{deg} / \mathrm{s}$ rate range was $<0.2 \%$ [11]. However, the nickel electroforming technology used for this device poses several drawbacks to improved device performance, including: 1) a fairly large ring to electrode gap spacing were achievable in the conventional electroplating process, which resulted in a small sense capacitance; although this could be overcome by more sophisticated technologies like LIGA, it is not desirable to use these because of cost considerations; 2) the thermal expansion coefficient of the silicon substrate is different than the sensor element, which was made out of nickel. Therefore as the temperature changes, the ring expands (or shrinks) more than the electrodes, which are attached to the substrate, causing the ring-to-electrode gap spacing to change; this in turn results in an increased temperature sensitivity of offset and scale factor; and 3) worse material properties of nickel, resulting in 
lower $Q$, and higher potential for creep, fatigue and long-term drift.

British Aerospace Systems and Equipment (BASE) has developed a micromachined single crystalline silicon ring gyroscope with a reported rms noise floor of $0.15^{\circ} / \mathrm{s}$ in a $30-\mathrm{Hz}$ bandwidth and an in-run drift of approximately $0.05 \%$ [12]. This device was fabricated through deep dry etching of $100-\mu \mathrm{m}$-thick silicon wafer which was then anodically bonded to a glass support wafer. Single crystal silicon ring structure will have the advantage of intrinsic high quality factor required for high performance vibratory gyroscopes. However, the Young's modulus of single crystal silicon varies with crystallographic orientation around the ring structure with a "Cos $4 \theta$ " dependence. For a uniform circular ring, this anisotropy will cause the sense and drive mode resonance frequencies to be different. This split in the frequency is significant and reduces the sensitivity if not compensated. Modulation of the ring width in a $\operatorname{Cos} 4 \theta$ manner can be used to compensate this anisotropy [13].

\section{The High Aspect-Ratio PRG}

To further improve the performance and overcome the shortcomings of the nickel vibrating ring gyroscope, the authors have developed a high aspect-ratio polysilicon vibrating ring gyroscope structure fabricated through the HARPSS MEMS Technology. The single wafer, all-silicon HARPSS process has been described in details in [8]. Using this technology, the vibrating ring and support springs are created by refilling $80 \mu \mathrm{m}$ deep dry-etched trenches with polysilicon deposited over a sacrificial LPCVD oxide layer. Each sense electrode is made out of low-resistivity single-crystal silicon island that is protected on the sides by deep refilled trenches and is released at the bottom from the substrate by means of an $\mathrm{SF}_{6}$ dry-release that is carried out entirely in a deep reactive ion etching (DRIE) system. The single-crystal silicon islands are all anchored on top to a supporting polysilicon layer, which is anchored through an isolating nitride layer to the substrate. The polysilicon ring is anchored in the middle to the substrate and is suspended using the support springs over an etch pit that is created through $\mathrm{SF}_{6}$ dry etching of silicon substrate in a DRIE system. Fig. 3 shows a SEM picture of a $1.7 \times 1.7 \mathrm{~mm}^{2} \mathrm{PRG}$. The ring is $1.1 \mathrm{~mm}$ in diameter and the diameter of the support post is $120 \mu \mathrm{m}$. The width of the ring and support springs is $4 \mu \mathrm{m}$. Sixteen electrodes are evenly located around the structure; they are approximately $60 \mu \mathrm{m}$ tall and $150 \mu \mathrm{m}$ long and are separated from the ring by a $1.4 \mu \mathrm{m}$ capacitive air-gap. Fig. 4 shows an SEM view of a $60-\mu \mathrm{m}$-tall electrode separated from the ring by $1.4 \mu \mathrm{m}$ capacitive gap in a prototype device.

This technology provides several important features that are required for high-performance MEMS microgyroscopes. First, it allows the polysilicon ring structure and the surrounding electrodes to be made hundreds of microns thick using DRIE technique. Second, since the ring to sense-electrode gap spacing is defined by the thickness of the sacrificial layer, it can be reduced to submicron level; these two factors together will significantly increase the sense capacitance and hence the device output signal. Third, the structural material is polysilicon which has a high quality factor and an orientation independent Young's

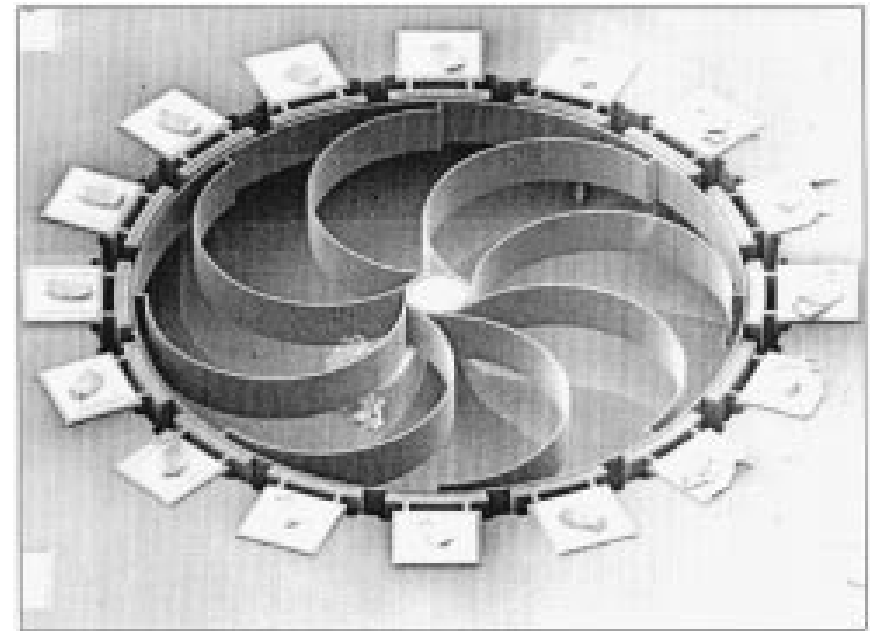

Fig. 3. SEM view of a prototype $1.7 \mathrm{~mm} \times 1.7 \mathrm{~mm}$ HARPSS PRG. The ring is $80-\mu \mathrm{m}$ thick and $1.1 \mathrm{~mm}$ in diameter; the diameter of the support post is 120 $\mu \mathrm{m}$.

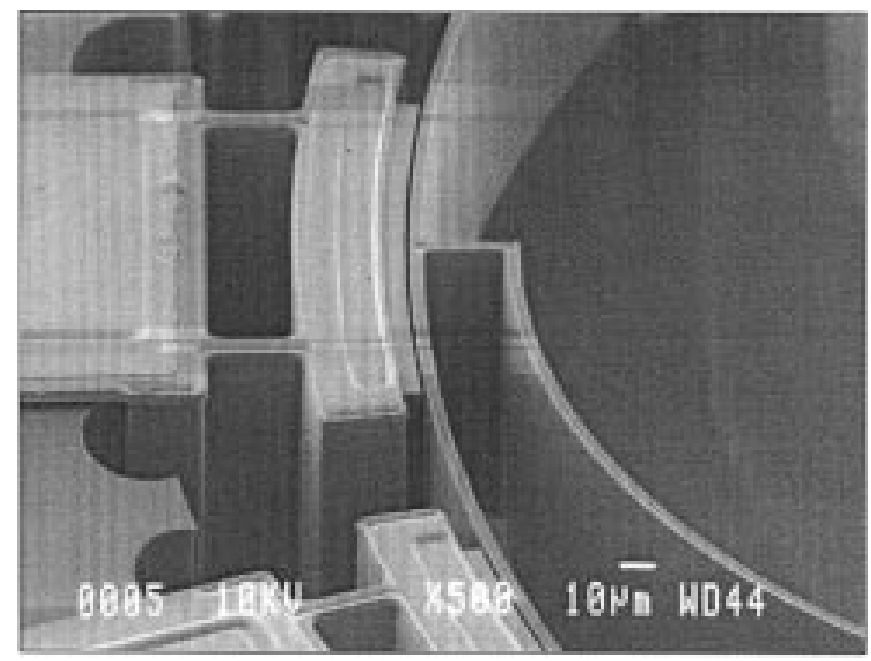

Fig. 4. Close-up of a $60-\mu \mathrm{m}$-tall electrode separated from the ring by $1.4 \mu \mathrm{m}$ capacitive gap in a HARPSS PRG

modulus. Fourth, the all-silicon feature of this technology improves long-term stability and temperature sensitivity. These features play an instrumental role in allowing the performance of the vibrating ring gyroscopes to be improved by orders of magnitude as indicated in the following analysis.

\section{SENSITIVITY AND NoISE ANALYSIS}

Vibration of the ring gyroscope causes the ring-to-electrode gap to vary as a function of time and position along the electrode. In order to vibrate the ring in its first flexural mode that has two nodal diameters, a sinusoidal electrostatic drive force is applied through the surrounding electrode located at a reference zero degree point. The ideal vibrating ring structure has two identical flexural modes that have equal resonant frequencies. Each sense electrode incorporates a low-input capacitance unity-gain buffer amplifier (source follower) to sense the small capacitance changes between the ring and the pickoff electrode due to ring vibration. Fig. 5 shows the detection scheme used 

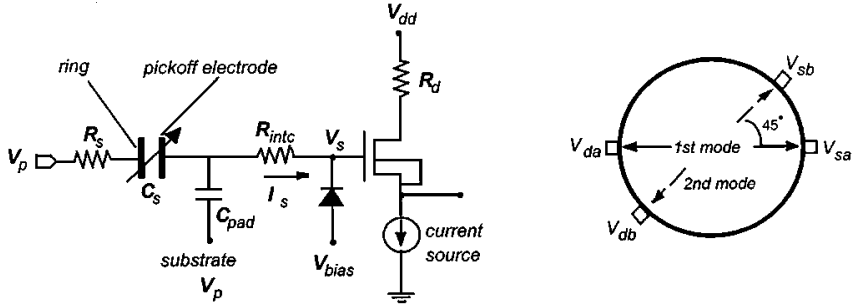

Fig. 5. Detection scheme used to sense ring vibration.

to sense ring vibrations. The ring is set at a polarization voltage $V_{p}$ to provide the bias for capacitive detection and to avoid frequency doubling of the drive force. The input bias voltage of the unity-gain buffer amplifier, $V_{\text {bias }}$, is set through an isolated minimum geometry diode [10]. Two pickoff electrodes, located at $0^{\circ}$ and $45^{\circ}$ are used to sense the ring vibration while two electrodes located directly across, at $180^{\circ}$ and $225^{\circ}$, are used to drive the ring (Fig. 5). Each drive electrode is driven by an ac drive voltage $\left(v_{d a}\right.$ and $\left.v_{d b}\right)$ which combine with the polarization voltage $V_{p}$. The ac voltages developed at the sense electrodes, $v_{s a}$ and $v_{s b}$, are generated by the vibration of the ring.

For each sense electrode, $V_{s}=V_{\text {bias }}+v_{s}$, where $V_{\text {bias }}$ is the bias voltage applied through the input diode and $v_{s}$ is the ac sense voltage. $I_{s}$, the current flowing through the combination of the sense electrode capacitance $\left(C_{s}\right)$ and the capacitance associated with the output pad to the substrate $\left(C_{\text {pad }}\right)$ (substrate is assumed to be at the polarization voltage $V_{p}$ ) is injected into the gate of the source follower and can be expressed by

$$
I_{s}=\frac{d}{d t}(Q)=\frac{d}{d t}\left[\left(C_{d}+\delta c_{d}+C_{\mathrm{pad}}\right)\left(V_{p}-V_{\mathrm{bias}}-v_{s}\right)\right]
$$

where $C_{d}$ and $\delta c_{d}$ are the average and the time varying components of the ring to sense electrode capacitance $\left(C_{s}\right)$, respectively. $R_{s}$ is the series resistance associated with the path connecting the point where $V_{p}$ is applied to the ring itself; due to the high doping level of polysilicon, this resistance is negligible compared to the impedance of the sense capacitance, and for simplifying purposes it is assumed to be zero. If the input capacitance of the buffer amplifier to ground is $C_{\text {inp }}$, one can find an expression for $v_{s}$ as [14]

$$
v_{s} \approx \frac{\delta c_{d}}{\left(C_{d}+C_{\mathrm{pad}}+C_{\mathrm{inp}}\right)}\left(V_{p}-V_{\mathrm{bias}}\right)
$$

Expression (3) states that in order to get higher sensitivity, the amount of change in ring to sense electrode capacitance due to the ring vibration should be maximized while the total sum of rest and parasitic capacitances $\left(C_{d}+C_{\text {pad }}+C_{\text {inp }}\right)$ should be minimized. So, a buffer amplifier with very small input capacitance must be used and the output pad capacitance to the substrate should be minimized. The electrode capacitance can be expressed by (to a good approximation)

$$
C_{s n}=\int_{\theta_{n}-\left(\Delta \theta_{n} / 2\right)}^{\theta_{n}+\left(\Delta \theta_{n} / 2\right)} \frac{\varepsilon \cdot h}{\left(d_{0}+\Delta d\right)} \cdot r \cdot d \theta
$$

where

$d_{0} \quad$ equilibrium gap spacing;

$\Delta d$ change in the gap due to vibration;

$h$ height of the structure;

$r$ radius of the ring;

$\Delta \theta$ angle faced by each electrode.

One can simplify (4) by using the Taylor series expansion

$$
\left(1+\frac{\Delta d}{d_{0}}\right)^{-1} \cong 1-\frac{\Delta d}{d_{0}}+\left(\frac{\Delta d}{d_{0}}\right)^{2}-\cdots, \text { for } \frac{\Delta d}{d_{0}} \ll 1
$$

to obtain

$$
\begin{aligned}
C_{s n}= & \frac{\varepsilon \cdot h \cdot r}{d_{0}} \\
& \cdot\left\{\int_{\theta_{n}-\left(\Delta \theta_{n} / 2\right)}^{\theta_{n}+\left(\Delta \theta_{n} / 2\right)}\left(d \theta-\frac{\Delta d}{d_{0}} \cdot d \theta+\left(\frac{\Delta d}{d_{0}}\right)^{2} \cdot d \theta\right)\right\} .
\end{aligned}
$$

As mentioned earlier, the ring vibrates in elliptically shaped mode patterns that have two node lines. In polar coordinates, $\Delta d=-\left(q_{1} \Phi_{r 1}+q_{2} \Phi_{r 2}\right)$, where the radial mode shape functions $\Phi_{r 1}$ and $\Phi_{r 2}$, for the ring portion of the sensor structure alone, are given by the expressions [10]

$$
\begin{aligned}
& \Phi_{r 1}=\cos \left(2 \theta-2 \theta_{0}\right) \\
& \Phi_{r 2}=\sin \left(2 \theta-2 \theta_{0}\right)
\end{aligned}
$$

$\theta_{0}$ is the location of the anti-node of the normal mode. In order to simplify the math, we can assume that $\theta_{0}$ is equal to zero; also for the sense electrode located at the $45^{\circ}$ position, $\Phi_{r 2}$ would be much larger than $\Phi_{r 1}$, allowing us to assume that $\Delta d \cong$ $-q_{2} \Phi_{r 2}\left(q_{2}\right.$ is the amplitude of the secondary vibration mode of the ring). Therefore, for the sense electrode located at $45^{\circ}$, we can further simplify (5) by neglecting the higher order terms to $C_{s n} \cong C_{d}+\delta c_{d}$, where

$$
\begin{aligned}
C_{d} & =\frac{\varepsilon \cdot h \cdot r}{d_{0}} \cdot \Delta \theta \\
\delta c_{d} & =\left[\frac{\varepsilon \cdot h \cdot r}{d_{0}^{2}} \cdot \sin (\Delta \theta)\right] \cdot q_{2} .
\end{aligned}
$$

If we combine (8) with (3), the following expression for the amplitude of the sense voltage developed at the $45^{\circ}$ pickoff can be obtained:

$$
v_{s} \cong \frac{\left(V_{p}-V_{\text {bias }}\right)}{\left(C_{d}+C_{\text {pad }}+C_{\text {inp }}\right)} \cdot \frac{\varepsilon \cdot h \cdot r \cdot \sin (\Delta \theta)}{d_{0}^{2}} \cdot q_{2} .
$$

In this equation, $q_{2}$ is the vibration amplitude of the sense mode $\left(q_{\text {sense }}\right)$ and is determined by the vibration amplitude of the drive mode, $q_{\text {drive }}$, through the rotation sensitivity of the ring structure given by (1). By combining (9) with (1), the following general expression for open loop sensitivity of the sensor is resulted:

$$
v_{s} \cong \frac{4 A_{g}\left(V_{p}-V_{\text {bias }}\right)}{\left(C_{d}+C_{\text {pad }}+C_{\text {inp }}\right)} \cdot \frac{\varepsilon \cdot h \cdot r \cdot \sin (\Delta \theta)}{d_{0}^{2}} \cdot \frac{\Omega_{z}}{\omega_{0}} \cdot Q \cdot q_{\text {drive }} .
$$


The minimum detectable rotation rate for the vibrating shell structure, $\Omega_{z(\min )}$, is directly proportional to the minimum detectable sense voltage, $v_{s(\min )}$, which is determined by the input referred noise of the interface circuit and the noise voltage developed at the sense node due to Brownian motion of the structure. The Brownian noise of the structure will be discussed in the next section and is assumed to be zero in the electronic noise floor calculations. If we assume that the noise from the output buffer, $V_{n}$, has a white spectrum near the flexing frequency of the ring and the bandwidth of the detection circuit is $B W$, then the minimum detectable rotation rate limited by electronic noise only (electronic noise floor) can be expressed by (11) shown at the bottom of the page.

Equation (11) suggests that in order to reduce the electronic noise floor of the ring gyroscope, one has to

- reduce the sense electrode gap spacing $\left(d_{0}^{2}\right)$;

- increase the height $(h)$ and radius $(r)$ of the structure as well as angle faced by each electrode $(\Delta \theta)$;

- increase the quality factor of the structure $(Q)$;

- reduce the natural resonance frequency of the structure $\left(\omega_{0}\right)$.

- minimize the input referred equivalent noise of the detection circuit $\left(V_{n}\right)$;

- minimize the total amount of the rest capacitances attached to the output node $\left(C_{d}+C_{\text {pad }}+C_{\text {inp }}\right)$;

- increase the drive amplitude ( $\left.q_{\text {drive }}\right)$;

- increase the polarization voltage $\left(V_{p}\right)$.

The vibration amplitude of the ring gyroscope, $q_{\text {drive }}$, should be set as large as possible to increase the sensitivity (stronger Coriolis forces will be resulted by increasing the amplitude of vibration in the drive mode $q_{\text {drive }}$ ), but it must be kept typically below $10 \%$ of the equilibrium gap spacing of the drive electrode to avoid the nonlinear effects of the electrostatic force. The polarization voltage cannot be increased indefinitely as $V_{p}$ has to be less than the pull-in voltage of the structure. The resonance frequency of the structure should be lowered for improved sensitivity; however, it must be kept above the environmental noise frequencies to make the sensor immune against spurious vibrations $(>2 \mathrm{kHz})$. However, a decrease in the resonance frequency of the structure will increase the Brownian noise floor of the device.

\section{A. Brownian Noise Floor}

Brownian motion of the proof mass caused by molecular collisions from the surrounding environment represents the fundamental limiting noise component of a sensor structure. By Nyquist's Relation, the spectral density of the fluctuating force related to any mechanical resistance $R$ is given by [15]

$$
F_{n}=\sqrt{4 k_{B} T R} \mathrm{~N} / \sqrt{\mathrm{Hz}}
$$

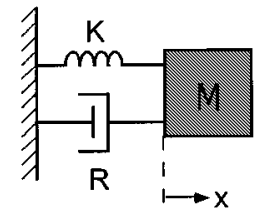

(a)

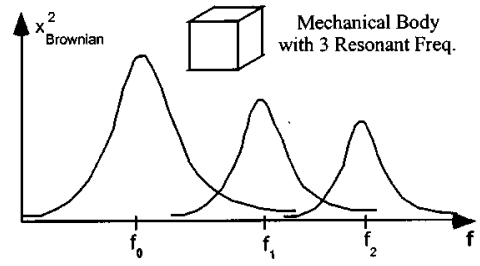

(b)
Fig. 6. (a) Second order mass-spring system. (b) Spectral density of displacement of a mechanical element with three resonant frequencies due to Brownian motion.

where $k_{B}$ is Boltzmann's constant $\left(1.38 \times 10^{-23} \mathrm{~J} / \mathrm{K}\right)$ and $T$ is the absolute temperature. For a second order mass-springdamper system shown in Fig. 6(a), the Brownian noise displacement corresponding to the fluctuating force related to a mechanical resistance $R$ becomes

$$
\left|x_{\text {Brownian }}\right|=\sqrt{4 k_{B} T R} \cdot G(f) \mathrm{m} / \sqrt{\mathrm{Hz}}
$$

where $G(f)$ is the displacement to force transfer function of the second-order mass-spring-damper system

$$
G(f)=\frac{K^{-1}}{\sqrt{\left[1-\left(\frac{f}{f_{0}}\right)^{2}\right]^{2}+\left(\frac{f}{Q f_{0}}\right)^{2}}}
$$

$K$ is the generalized stiffness of the structure, $R=K /\left(Q \omega_{0}\right)$, $Q=\sqrt{K M} / R=\omega_{0} M / R$, and $\omega_{0}=\sqrt{K / M} . M$ is the generalized mass of the vibration mode, $\omega_{0}$ is the resonance frequency of the vibration mode, and $Q$ is the quality factor. It should be noted that (12) can be derived directly from the equipartition theorem which states that any energy storage mode of a system in thermal equilibrium has an average energy of $\frac{1}{2} k_{B} T$ [15]. The mean square Brownian noise displacement of a mass-spring-damper system for a given resonance mode is expressed by

$$
\frac{1}{2} K\left\langle x^{2}\right\rangle=\frac{1}{2} k_{B} T
$$

where $\left\langle x^{2}\right\rangle$ is the integral sum of $|x|^{2}$ over all frequencies. Using (15) and considering the fact that for a mass-spring-damper system $|x|=F_{n} . G(f)$ in which $F_{n}$ is the white noise force, integration of $|x|^{2}$ over range of all frequencies $(0, \infty)$ will result in (12) [16].

The displacement noise $x_{\text {Brownian }}$ peaks at the resonance frequency and becomes $Q$ times larger than below resonance values, as shown in Fig. 6(b)

$$
\text { for } \omega=\omega_{0} \Rightarrow\left|x_{\text {Brownian }}\right|=\sqrt{\frac{4 k_{B} T Q}{M \omega_{0}^{3}}}
$$

$$
\Omega_{z \text { (electronic) })} \cong \frac{\left(C_{d}+C_{\text {pad }}+C_{\text {inp }}\right)}{4 A_{g}\left(V_{p}-V_{\text {bias }}\right)} \cdot \frac{d_{0}^{2}}{\varepsilon \cdot h \cdot r \cdot \sin (\Delta \theta)} \cdot \frac{\omega_{0}}{Q} \cdot \frac{1}{q_{\text {drive }}} V_{n} \cdot \sqrt{B W}
$$


For a ring gyroscope with matched drive and sense resonance modes, the output displacement response of the sense mode to an input rotation rate of $\Omega_{z}$ due to the Coriolis force of $f_{\text {Coriolis }}=4 A_{g} \Omega_{z} M \omega_{0} q_{\text {drive }}$ is expressed by

$$
x_{s}=4 A_{g} \Omega_{z} M \omega_{0} q_{\text {drive }} \cdot G(f) .
$$

Therefore, the signal-to-thermal noise ratio at any frequency in a bandwidth of BW is then expressed by

$$
\left|\frac{x_{s}}{x_{\text {Brownian }}}\right|=\frac{4 A_{g} \Omega_{z} M \omega_{0} d_{\text {drive }}}{\sqrt{4 k_{B} T \frac{\omega_{0} M}{Q}} \cdot \sqrt{B W}} .
$$

By $\left|x_{s} / x_{\text {Brownian }}\right|=1$, we can find an expression for the Brownian noise floor of the ring gyroscope in a bandwidth of BW

$$
\Omega_{z(\text { Brownian }))}=\frac{1}{4 A_{g} q_{\text {drive }}} \cdot \sqrt{\frac{4 k_{B} T}{\omega_{0} M Q}} \cdot \sqrt{B W} \mathrm{rad} / \mathrm{s} .
$$

Therefore, in order to reduce the Brownian noise floor of a ring gyroscope:

- the generalized mass of the structure $(M)$ and its resonance frequency $(\omega)$ should be increased;

- the amplitude of the drive mode should be increased $\left(q_{\text {drive }}\right)$;

- the sensor structure should have a high quality factor $(Q)$.

Since the Brownian and the electronic noise are uncorrelated, the overall noise floor of the gyroscope is then given by

$$
\Omega_{z(\min )}=\sqrt{\Omega_{z(\text { electronic })}^{2}+\Omega_{z(\text { Brownian })}^{2}} .
$$

\section{B. Resonance Frequency of the Ring Gyroscope}

The natural frequency for the radial-circumferential flexural modes of a cylindrical shell with a radius $r$ and a shell thickness $t$ is given by [17]

$$
f_{i}=\frac{1}{2 \pi} \cdot \frac{1}{\sqrt{12}} \cdot \frac{t}{r^{2}} \cdot \frac{i\left(i^{2}-1\right)}{\sqrt{\left(i^{2}+1\right)}} \cdot \sqrt{\frac{E}{\rho}}, \quad i=2,3,4, \cdots
$$

where $E$ is the Young's modulus and $\rho$ is the density of the shell material. Natural frequencies of the flexural modes are independent of the height of the shell structure. Using (21), it is possible to approximately calculate the flexural resonance frequency of the ring gyroscope. Since $f_{i}=(1 / 2 \pi) \sqrt{k_{1} / M}$ and $M$ (mass) $\propto r$, we can see that $k_{i} \propto\left(1 / r^{3}\right)$. In case of a ring gyroscope in which the radius of each supporting spring is approximately equal to 0.43 of the radius of the ring itself, flexural stiffness of each spring can be calculated to be 6.4 times larger than the stiffness of the ring shell without any springs attached to it (note that two springs in parallel make a full ring; therefore, the stiffness of each semicircular spring is half of the stiffness of a circular ring). Therefore, the overall stiffness for the flex-
TABLE I

SPECIFICATIONS AND CALCULATED PERFoRmanCE OF THE HARPSS POLYSILICON VIBRATING RING GYROSCOPE

\begin{tabular}{c|c}
\hline \hline Design Parameter & Target Value \\
\hline Material Quality Factor & 20,000 \\
\hline Ring Diameter & $1.1 \mathrm{~mm}$ \\
\hline Ring and Springs Width & $4 \mu \mathrm{m}$ \\
\hline Height of the Ring Structure & $80 \mu \mathrm{m}$ \\
\hline Support Post Diameter & $120 \mu \mathrm{m}$ \\
\hline Support Spring Diameter & $470 \mu \mathrm{m}$ \\
\hline Sense Electrode Gap Spacing & $1.4 \mu \mathrm{m}$ \\
\hline Height of Each Electrode & $60 \mu \mathrm{m}$ \\
\hline Resonant Frequency & $29 \mathrm{kHz}$ \\
\hline Interface Circuit Input Noise $\left(\mathrm{V}_{\mathrm{n}}\right)$ & $1 \mu \mathrm{V} /(\mathrm{Hz})^{0.5}$ \\
\hline Parasitic Capacitance $\left(\mathrm{C}_{\text {device }}+\mathrm{C}_{\mathrm{pad}}\right)$ & $500 \mathrm{fF}$ \\
\hline Polarization Voltage $\left(\mathrm{V}_{\mathrm{p}}\right)$ & $7 \mathrm{~V}$ \\
\hline Drive amplitude & $0.15 \mu \mathrm{m}$ \\
\hline Brownian Noise Floor & $0.01 \mathrm{deg} / \mathrm{sec} /(\mathrm{Hz})^{0.5}$ \\
\hline Electronic Noise Floor & $8 \times 10^{-3} \mathrm{deg} / \mathrm{sec} /(\mathrm{Hz})^{0.5}$ \\
\hline Min. Detectable Signal (10Hz BW) & $\mathbf{0 . 0 4} \mathrm{deg} / \mathrm{sec}$ \\
\hline \hline
\end{tabular}

ural mode of the ring gyroscope with eight springs will be 7.4 times the stiffness of a ring without springs. If we consider the effective mass of the spring to be one third of its actual mass, then the natural frequency for the first flexural mode of the ring can be estimated by $(i=2$ in (21))

$$
f_{\text {ring gyro }} \cong \frac{\sqrt{3}}{2 \pi} \cdot \frac{t}{r^{2}} \cdot \sqrt{\frac{E}{\rho}} .
$$

If we assume that the Young's modulus of polysilicon is approximately equal to $E=150 G P a=1.5 \times 10^{12}$ dynes $/ \mathrm{cm}^{2}$ and its density is equal to $\rho_{S i}=2.328 \mathrm{~g} / \mathrm{cm}^{3}$, for a ring gyroscope with a ring diameter of $1.1 \mathrm{~mm}$ and a ring width $(t)$ of $4 \mu \mathrm{m}$, the resonance frequency of the first flexural mode can be calculated to be equal to $f_{\text {ring gyro }} \cong 29 \mathrm{kHz}$. Care must be taken in using (22) since it has been derived based on the specific dimensions of the polysilicon ring gyroscope described in this paper. Table I summarizes the design parameters and the expected resolution for a $1.1 \mathrm{~mm}$ in diameter, $80 \mu \mathrm{m}$ tall HARPSS polysilicon ring gyroscope.

\section{Test Results}

Prototypes of $80 \mu \mathrm{m}$ thick HARPSS polysilicon ring gyroscopes were tested under vacuum for open loop operation. The sensor chip was connected in hybrid format to an NMOS sourcefollower buffer chip, both mounted on a 24-pin ceramic DIP package as shown in Fig. 7, to measure ring vibrations. The low-impedance output signal of this buffer chip was then amplified off-chip by a factor of 100 using discrete amplifiers on a printed circuit board (PCB). This PCB was installed on the rate table, inside the vacuum chamber and its communication with the drive and control circuitry located outside the chamber was made through a set of slip rings. Drive and control circuitry needed for tuning and open loop operation of the ring gyroscope were implemented off chip using discrete components. Part of the electronics drives the first mode of the structure into resonance and keeps the amplitude of vibration constant. This is 


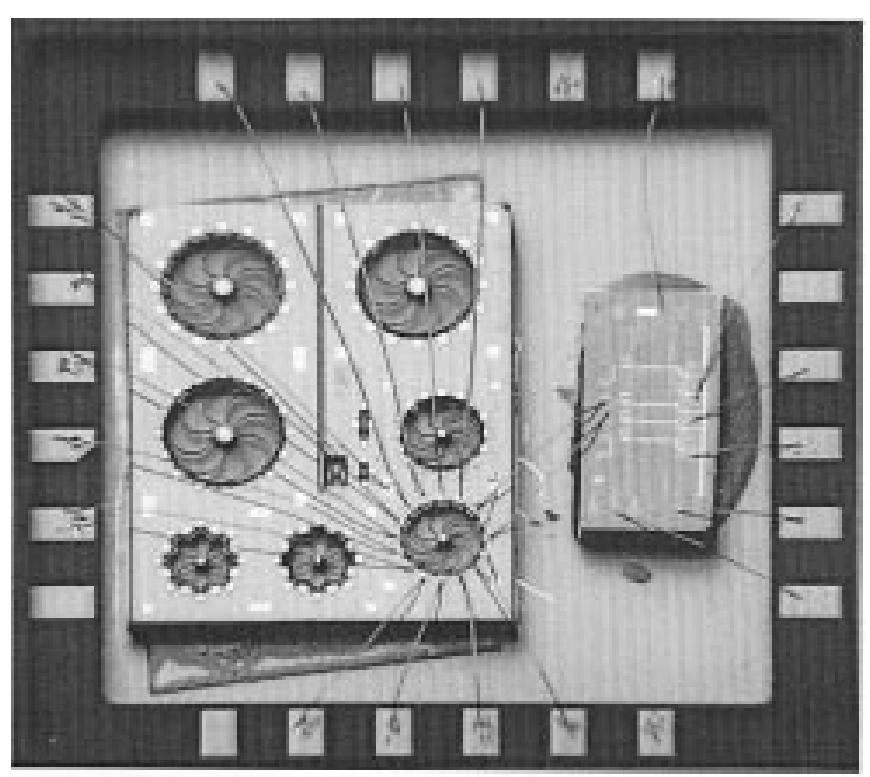

Fig. 7. Hybrid attachment of the ring gyroscope and the unity-gain buffer IC chip in a 24-pin ceramic DIP package.

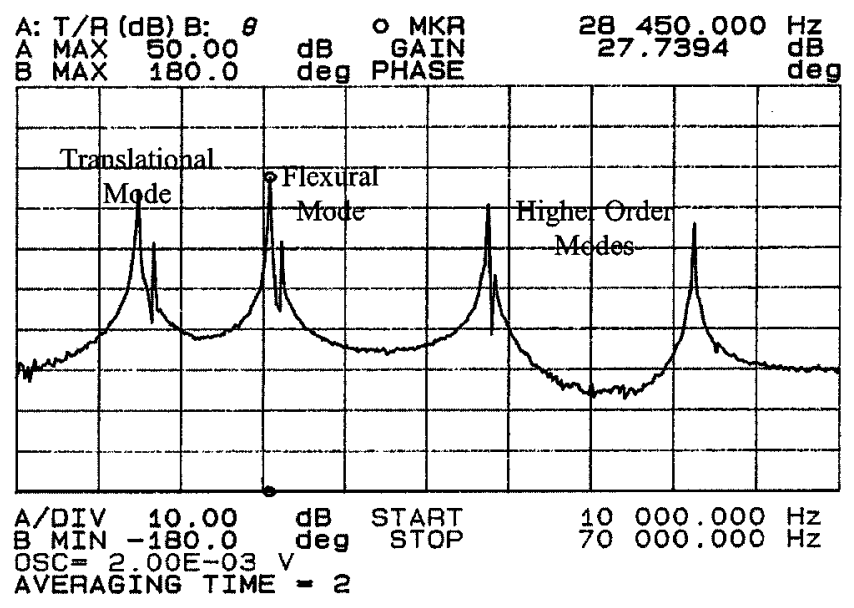

Fig. 8. Frequency response of a prototype HARPSS polysilicon ring gyroscope, showing several vibration modes of the structure.

done by using a phase locked loop (PLL), which continuously forces the frequency of a VCO to track the resonance frequency of the structure. Control electronics will then develop the proper control voltage $\left(v_{d a}\right)$ by use of a synchronous demodulator to keep the amplitude of vibration constant.

Fig. 8 shows the measured frequency response of an $80 \mu \mathrm{m}$ thick, $4 \mu \mathrm{m}$ wide, $1.1 \mathrm{~mm}$ in diameter HARPSS PRG, showing various vibration modes of the structure. In this figure, some peaks are in pair. Each pair represents a degenerate mode, such as translational and flexural modes, and frequencies of the two degenerate modes are slightly different due to imperfection or deformation of the ring structure (frequencies are equal in an ideal uniform ring). Electronic balancing will pull the two frequencies together and make them equal through the action of electrostatic springs. Vibration modes of the polysilicon ring gyroscope were also obtained through finite element method (FEM) simulations in ANSYS. Table II summarizes the results obtained from the FEM normal mode analysis of the sensor structure. Simulation results are in good agreement with the
TABLE II

ANSYS SIMULATION RESUlts (shell63) FOR NORMAL MODE ANALYSIS OF A PRG WITH FOLLOWING DIMENSIONS: $1.1 \mathrm{~mm}$ IN DIAMETER; $80 \mu \mathrm{m}$ TALL; 4 $\mu \mathrm{m}$ WIDE; $120 \mu \mathrm{m}$ IN DIAMETER SUPPORT POST

\begin{tabular}{c|c}
\hline \hline Vibration Mode & Frequency (kHz) \\
\hline Torsional & 10.95 \\
\hline $\begin{array}{c}\text { In-plane translational } \\
\text { "two degenerate modes" }\end{array}$ & 20.18 \\
\cline { 2 - 2 } $\begin{array}{c}\text { Flexural (2 node lines) } \\
\text { "two degenerate modes" }\end{array}$ & 20.18 \\
\cline { 2 - 2 } Higher order flexural (3 node lines) & 29.28 \\
\cline { 2 - 2 } "two degenerate modes" & 43.08 \\
\hline Higher order flexural (4 node lines) & 43.08 \\
\hline \hline
\end{tabular}

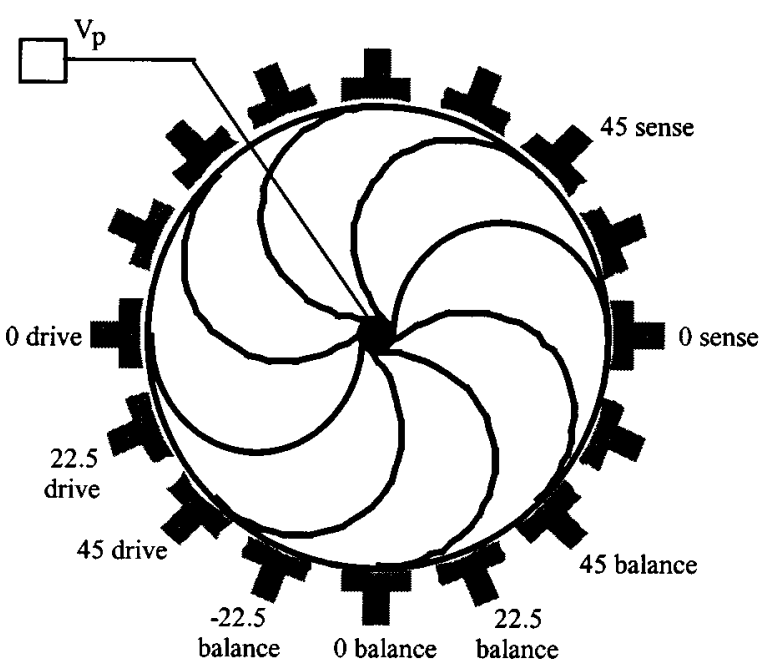

Silicon substrate

Fig. 9. Electrode representation for the ring gyroscope.

test results. The torsional and out-of-plane modes cannot be excited with current electrode configuration. ANSYS calculated a frequency of $20.18 \mathrm{kHz}$ for the in-plane translational modes of the structure and $29.28 \mathrm{kHz}$ for the first flexural mode. The frequency of the first flexural mode of a prototype device is measured to be $28.3 \mathrm{kHz}$ (with $7 \mathrm{~V}$ bias applied to the ring) which agrees well with the calculated value of $29 \mathrm{kHz}$ as well as ANSYS simulation results.

It should be noted that bent-beam strain sensors were also fabricated using the HARPSS process to measure the residual stress in $80 \mu \mathrm{m}$ thick $4 \mu \mathrm{m}$ wide trench-refilled polysilicon beams. As reported in reference [8], the in-plane stress for thick polysilicon trench-refilled beams was measured to be virtually zero. This was expected for the trench-refill process in which the residual stress of counterfacing polysilicon layers on the trench sidewalls cancel out each other as the trench is refilled. The good agreement of the measured resonance frequencies with ANSYS simulation results is also another indication that the residual stress in trench-refilled polysilicon beams is negligible.

\section{A. Electronic Tuning}

Electronic tuning is an attractive feature of the vibrating ring gyroscope. Any frequency mismatch between the sense 


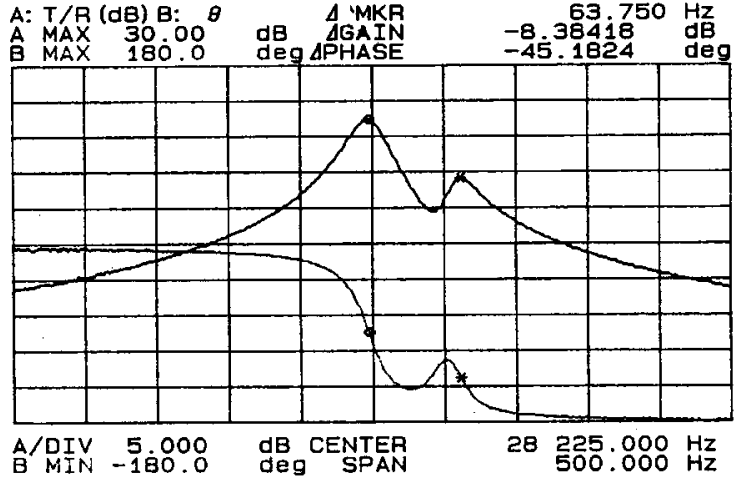

(a)

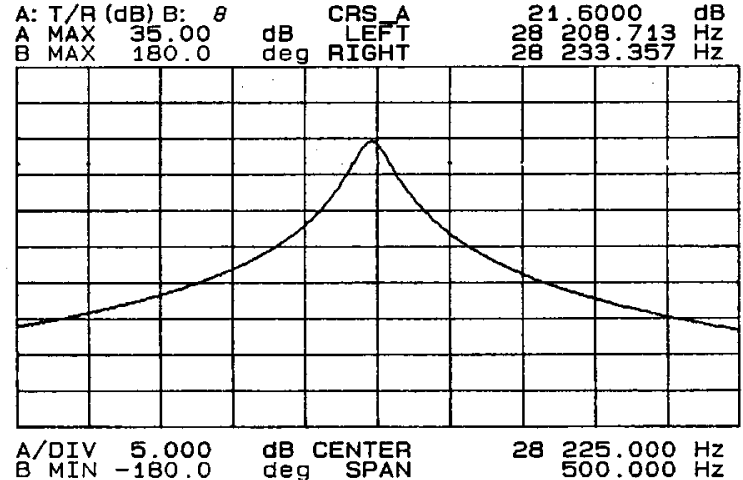

(b)

Fig. 10. Electronic balancing of the ring gyroscope. (a) Before balancing: Two flexural resonant peaks of a prototype ring gyroscope have different frequencies ( $\Delta f=63 \mathrm{~Hz}$ for $f_{0}=28 \mathrm{kHz}$ ). (b) After balancing: Two peaks merge together and the frequencies become equal.

and drive modes that occur during the fabrication process can be electronically compensated using the tuning electrodes around the structure. By matching the drive and sense mode frequencies, sensitivity is amplified by the quality factor of the structure. The tuning procedure for the ring gyroscope has been explained in details in reference [10] and is accomplished by applying dc voltages to two pairs of electrodes as shown in Fig. 9: one pair located at $22.5^{\circ}$ and $-22.5^{\circ}$, and the other pair located at $0^{\circ}$ and $45^{\circ}$ angles. The rest of the surrounding electrodes (except the $0^{\circ}$ sense and drive and $45^{\circ}$ sense electrodes) were grounded. Fig. 10(a) shows the two flexural resonance peaks of a prototype device before the electronic tuning, showing a frequency split of $63 \mathrm{~Hz}$. The frequencies of the two modes of a device can be apart by even a few hundred Hertz due to imperfection in the fabrication process and/or device structure as well as electrostatic stiffening effect of bias voltages applied to the surrounding electrodes. The balancing voltages required to null the $63 \mathrm{~Hz}$ of frequency split between the modes of this prototype single ring gyroscope were very small: $V \pm_{22.5}= \pm 0.9 \mathrm{~V}$ and $V_{0,45}=0 \mathrm{~V}$. After balancing, the two peaks merge together and the sense and drive mode frequencies become equal, as shown in Fig. 10(b). It is noteworthy that the balancing voltages needed to null 40 $\mathrm{Hz}$ of frequency split between the two modes of a prototype $20 \mu \mathrm{m}$ tall nickel gyroscope with a $6 \mu \mathrm{m}$ capacitive gap and a flexural frequency of $f_{0}=33 \mathrm{kHz}$ was $V \pm_{22.5}= \pm 15.5 \mathrm{~V}$ [10]. Small capacitive gaps $(1.4 \mu \mathrm{m})$ and tall electrodes have indeed shifted the tuning voltages down to CMOS acceptable levels, increasing the tuning capability. We have also been able to balance ring structures with up to $1 \mathrm{kHz}$ of frequency split $\left(f_{0}=28 \mathrm{kHz}\right)$ by applying less than 6 Volts tuning voltages to the balancing electrodes [14].

The quality factor $(Q)$ for the first flexural mode of this prototype device was measured to be 2000 after balancing. This is smaller than anticipated due to excessive anchor and bulk losses. Fig. 11 shows a typical anchor problem encountered in early designs of the poly ring gyro. Excessive undercut of the silicon substrate at the post causes the interface oxide layer between the substrate and structural poly to be exposed during the HF release and get etched away, which in turn will result in a soft anchor that dissipates energy. Solid posts significantly reduce dissipa-

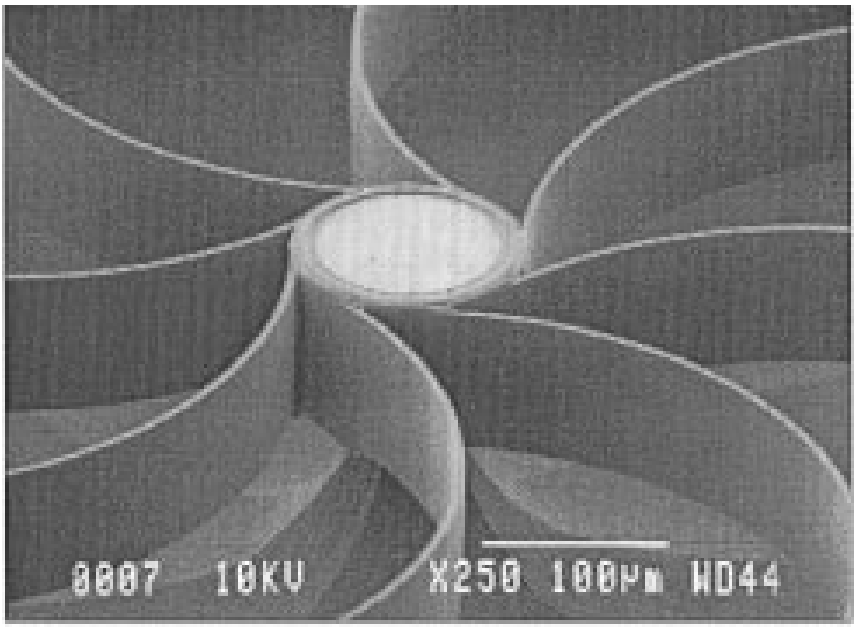

Fig. 11. SEM view of the anchor of a poly ring gyroscope. Excessive undercut of the silicon substrate at the post can result in a soft anchor point and dissipation of energy.

tion of energy through anchor losses. The highest $Q$ measured for the first flexural mode of the polysilicon ring gyroscope with modified solid postdesign has been 6000 under 1 mtorr vacuum [14]. Another possible source of dissipation of energy in trench-refilled polysilicon beams are voids and keyholes that can be generated during the polysilicon refill process, shown in Fig. 12. In order to avoid this problem, trenches with completely vertical (or slightly slanted inward) sidewall profile are needed and enough polysilicon needs to be deposited to completely refill trenches at the wide intersection points. Modified devices with solid post and voidless polysilicon beams are expected to have higher $Q$ in the range of 10000-20000 and hence higher sensitivity. $80 \mu \mathrm{m}$ tall, $300 \mu \mathrm{m}$ long clamped-clamped beam micromechanical resonators fabricated in this technology have shown quality factors as high as 85000 in 1 mTorr vacuum [8].

\section{B. Open-Loop Testing}

The block diagram of the drive and control circuitry needed for open loop operation of the polysilicon ring gyroscope is shown in Fig. 13. The circuitry was mounted on a bread-board 


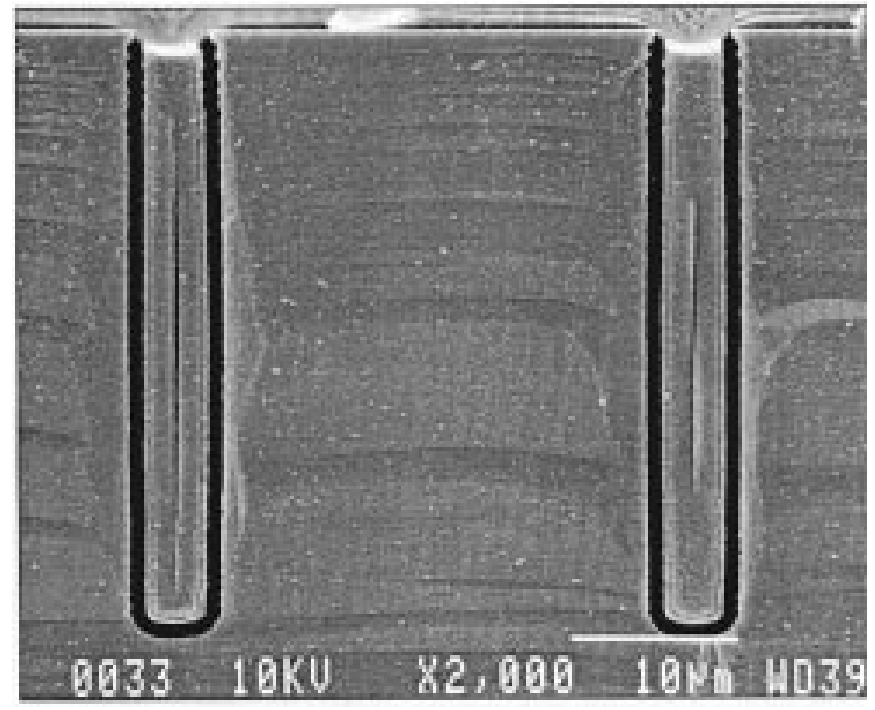

Fig. 12. Voids and keyholes generated during the polysilicon refill process can be potentially a source of dissipation of energy.

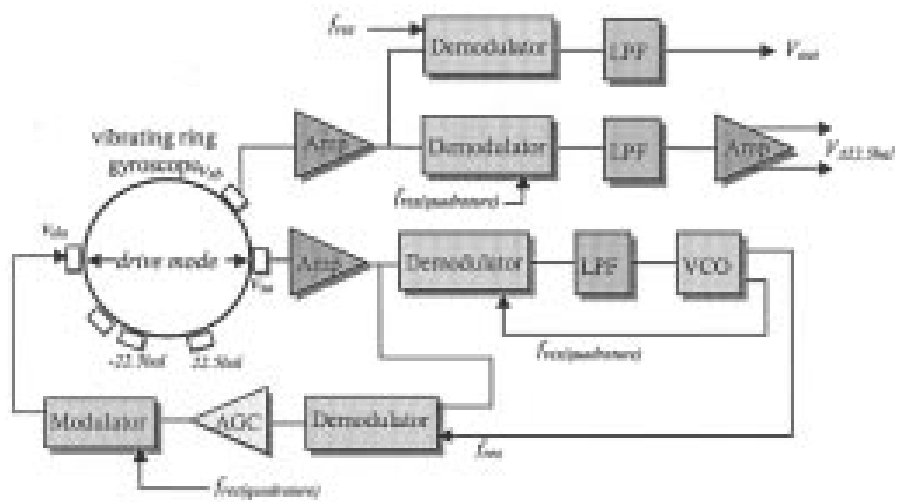

Fig. 13. Block diagram of the drive, readout and control circuitry for open loop operation of the polysilicon ring gyroscope.

outside of the vacuum chamber and consists of three main loops: The main control loop that is based on a phase locked loop oscillator circuit that locks into the resonance frequency of the gyroscope. This PLL circuit is based on the phase relationship between the drive force and the output displacement. At resonance, the vibrational displacement of the structure lags behind the applied drive force by $90^{\circ}$. This phase difference is measured using a balanced demodulator. The second loop, which is called the amplitude control loop, accurately control the amplitude of the reference vibration to minimize gain errors in the sensor output. So, the main and amplitude control loops operate together to resonate the ring structure into its first flexural mode and keep the vibration amplitude constant. Fig. 14 shows the waveforms from a prototype polysilicon ring gyroscope. The lower trace is the square wave drive signal applied to the $0^{\circ}$ drive electrode and the top trace is the output signal recorded from the $0^{\circ}$ sense electrode. The two waveforms have the anticipated $90^{\circ}$ phase difference. The typical amplitude of the drive signal is around 5-8 $\mathrm{mV}$ peak. Larger drive levels will cause distortion in the output signal (maximum displacement must be kept less than $10 \%$ of the air-gap for linear operation in electrostatic parallel plate actuators).

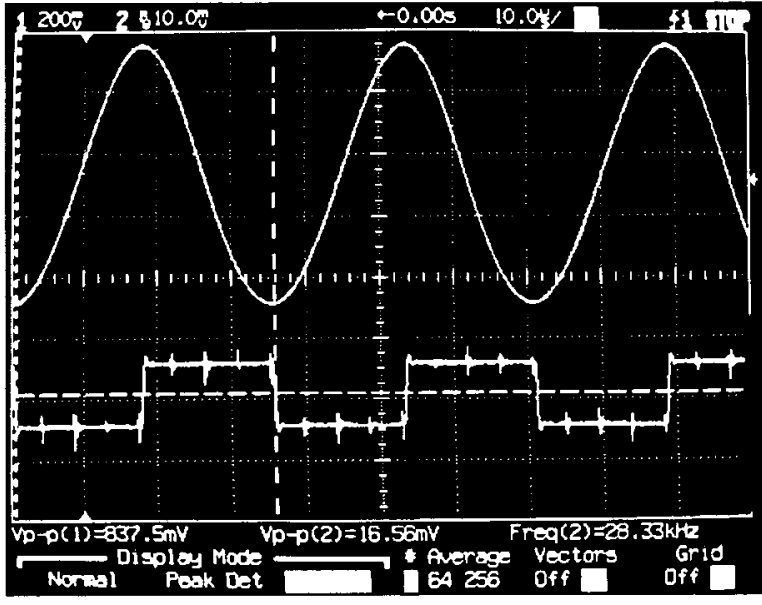

Fig. 14. Square-wave drive signal generated by the lock-in amplifier and the output signal from the gyroscope.

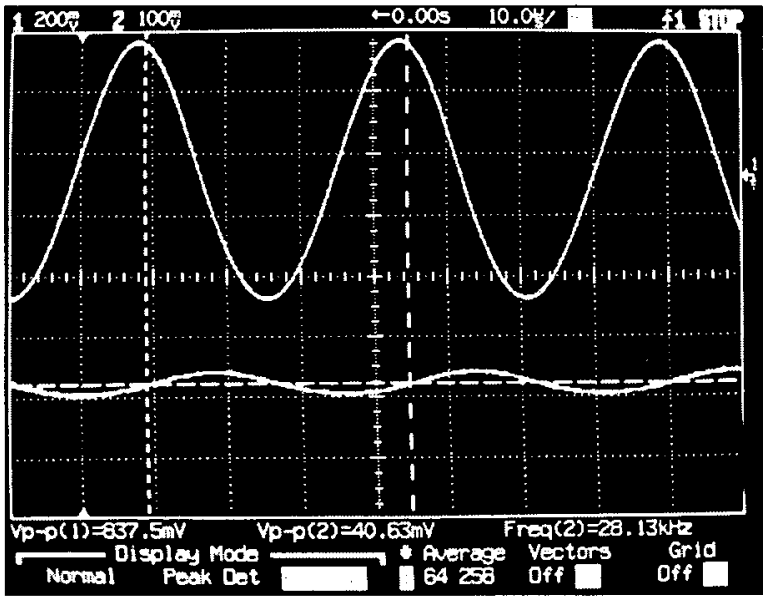

Fig. 15. ZRO of the gyroscope (bottom trace) after balancing. The top trace shows the output of the drive mode.

Once the circuit has locked to the flexural mode, the electronic tuning of the structure will be performed. The third loop, which is called the quadrature control loop, continuously adjusts one pair of balancing voltages located at $\pm 22.5^{\circ}$ to null out the quadrature signal, or the zero rate output (ZRO), which is in phase quadrature with the Coriolis-induced output signal. Issues related to each of these control loops have been discussed in details in [10]. For closed-loop operation of the sensor, an extra loop has to be used to force the output displacement to zero. Fig. 15 shows the zero rate output of a prototype ring gyroscope after balancing. The bottom trace is the $45^{\circ}$ sense signal and the top trace is the $0^{\circ}$ sense signal and the device was drive at $0^{\circ}$ drive. The quadrature phase difference can be verified in this picture.

The response of a prototype polysilicon ring gyroscope to input rotation rates up to $\pm 250 \mathrm{deg} / \mathrm{s}$ was measured in a bandwidth of $5 \mathrm{~Hz}$. Fig. 16 shows the response of the device to a $5 \mathrm{~Hz}$ sinusoidal input rotation rate with a peak-to-peak amplitude of $120 \mathrm{deg} / \mathrm{s}$. It should be noted that this measurement was carried out under very poor vacuum level and the quality factor was only about 250 which is an order of magnitude less than the actual $Q$ of the ring structure under high vacuum. This drop 


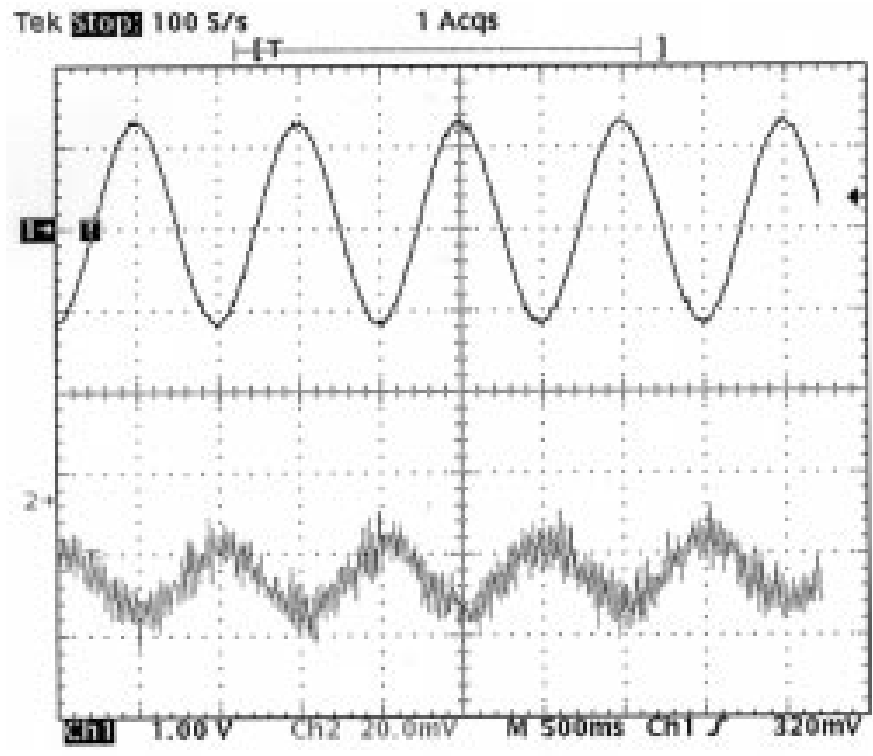

Fig. 16. Response of a prototype single ring gyroscope (lower trace) to a $5-\mathrm{Hz}$ sinusoidal rate with a peak-to-peak amplitude of $120 \mathrm{deg} / \mathrm{s}$.

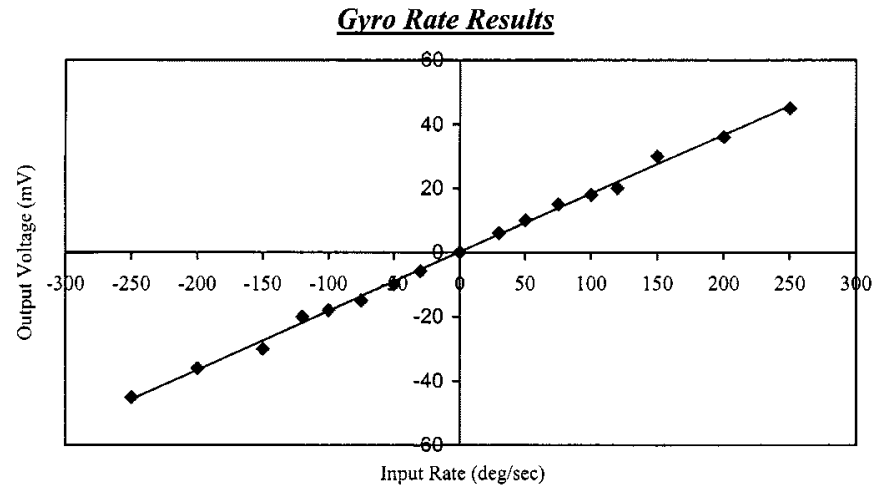

Fig. 17. Response of a prototype PRG to input rotation rates in a $5 \mathrm{~Hz} \mathrm{BW}$. The sensitivity of this device under poor vacuum $(Q \approx 250)$ was around 200 $\mu \mathrm{V} / \mathrm{deg} / \mathrm{s}$.

in the quality factor reduces the open loop output signal by a factor of 10 . Also, the output signal has been further reduced due to large amount of parasitic capacitance in the output sense node. The rest capacitance associated with each electrode in a single ring design has been measured to be around $500 \mathrm{fF}$, which agrees with calculated value. However, the input capacitance of the buffer chip along with the capacitance associated with the bonding pad on the IC chip and, the parasitic capacitance of the long wire-bonds used for hybrid connection have been measured to be around $1.5 \mathrm{pF}$ total. Therefore the total amount of the rest capacitance associated with each sense pad was around $2 \mathrm{pF}$ ! This large capacitance has degraded the output signal by an additional factor of 4 . Elimination of these two factors will increase the output signal by a factor of 40 .

Fig. 17 is the response of the sensor to input rotation rates in $5 \mathrm{~Hz}$ bandwidth. The sensitivity under very poor vacuum $(Q \approx 250)$ and large parasitics $(2 \mathrm{pF})$ was measured to be 200 $\mu \mathrm{V} / \mathrm{deg} / \mathrm{s}$. Another set of measurements was taken under im-

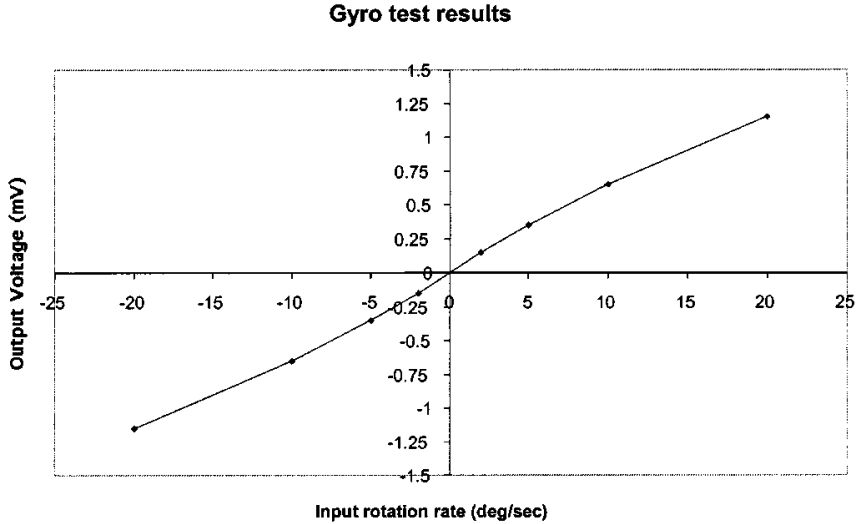

Fig. 18. Measured rate results obtained from a prototype PRG in a 5-Hz BW, range $= \pm 20 \% \mathrm{~s}(Q \approx 1200)$.

proved vacuum levels. The $Q$ of the device was measured to be approximately 1200 after balancing. Fig. 18 shows the response of the sensor to rotation rates in the range of $-20 \mathrm{deg} / \mathrm{s}$ to 20 $\mathrm{deg} / \mathrm{s}$. The resolution of the sensor was measured to be $\sim 1 \mathrm{deg} / \mathrm{s}$ in $1 \mathrm{~Hz}$ bandwidth. This resolution for a $Q$ of 1200 and parasitic capacitances of $2 \mathrm{pF}$ is in agreement with the theory. The resolution of the sensor is currently limited by the noise from the circuitry. Elimination of parasitic capacitances and improvement in $Q$ will reduce the resolution to $0.01 \mathrm{deg} / \mathrm{s}$ in $1 \mathrm{~Hz}$ bandwidth, which is the Brownian noise floor for the current design of the polysilicon ring gyroscope with a drive amplitude of $0.15 \mu \mathrm{m}$.

\section{CONCLUSION}

This paper presents the design, fabrication and testing of a high aspect-ratio $80 \mu \mathrm{m}$ thick, $1.1 \mathrm{~mm}$ in diameter polysilicon ring gyroscope fabricated using the HARPSS process. The single-crystal silicon electrodes were $60 \mu \mathrm{m}$ tall and the capacitive gaps were $1.4 \mu \mathrm{m}$. An open-loop sensitivity of $200 \mu \mathrm{V} / \mathrm{deg} / \mathrm{s}$ in a dynamic range of $\pm 250 \mathrm{deg} / \mathrm{s}$ was measured under low vacuum level for a prototype device tested in hybrid format. The resolution for a prototype sensor with a quality factor $(Q)$ of 1200 , drive amplitude of $0.15 \mu \mathrm{m}$, and sense-node parasitic capacitance of $2 \mathrm{pF}$ was measured to be less than $1 \mathrm{deg} / \mathrm{s}$ in $1 \mathrm{~Hz}$ bandwidth. Elimination of the parasitic capacitance and improvement in the quality factor of the ring structure will improve the resolution to $0.01 \mathrm{deg} / \mathrm{s} /(\mathrm{Hz})^{0.5}$.

\section{ACKNOWLEDGMENT}

The authors would like to thank Dr. M. Putty of General Motors for his contributions and help in testing and Prof. Y. Gianchandani of the University of Wisconsin for discussion on noise. They also wish to thank H. H. Chen, F. Kocer, and Dr. G. He for their valuable help in setting up the rate table and in electrical testing.

\section{REFERENCES}

[1] N. Yazdi, F. Ayazi, and K. Najafi, "Micromachined inertial sensors," Proc. IEEE, pp. 1640-1659, Aug. 1998.

[2] T. K. Tang et al., "A packaged silicon MEMS vibratory gyroscope for microspacecraft," in Proc. IEEE Micro Electro Mechanical Systems Workshop (MEMS '97), Japan, 1997, pp. 500-505. 
[3] M. Weinberg, J. Bernstein, S. Cho, A. T. King, A. Kourepenis, P. Ward, and J. Sohn, "A micromachined comb-drive tuning fork gyroscope for commercial applications," in Proc. Sensor Expo, OH, 1994, pp. 187-193.

[4] W. Geiger, B. Folkmer, J. Merz, H. Sandmaier, and W. Lang, "A new silicon rate gyroscope," in Proc. IEEE Micro Electro Mechanical Systems Workshop (MEMS '98), Heidelberg, Germany, 1998, pp. 615-620.

[5] K. Y. Park et al., "Lateral gyroscope suspended by two gimbals through high aspect ratio ICP etching," in Tech. Dig. 10th Int. Conf. on Solid-State Sensors and Actuators (Transducers '99), Sendai, Japan, June 1999, pp. 972-975.

[6] F. Ayazi and K. Najafi, "Design and fabrication of a high-performance polysilicon vibrating ring gyroscope," in Proc. IEEE Micro Electro Me chanical Systems (MEMS) Workshop, Heidelberg, Germany, Feb. 1998, pp. 621-626.

[7] F. Ayazi et al., "A high aspect-ratio polysilicon vibrating ring gyroscope," in Proc. Solid-State Sensor and Actuator Workshop, Hilton Head, SC, June 4-8, 2000, pp. 289-292.

[8] F. Ayazi and K. Najafi, "High aspect-ratio combined poly and silicon crystal silicon (HARPSS) MEMS technology," J. Microelectromech. Syst., vol. 9, pp. 288-294, Sept. 2000.

[9] M. W. Putty and K. Najafi, "A micromachined vibrating ring gyroscope," in Proc. Digest, Solid-State Sensors and Actuators Workshop, Hilton Head, SC, June 1994, pp. 213-220.

[10] M. W. Putty, "A micromachined vibrating ring gyroscope," Ph.D. dissertation, Univ. Michigan, Mar. 1995.

[11] D. R. Sparks, S. R. Zarabadi, J. D. Johnson, Q. Jiang, M. Chia, O. Larsen, W. Higdon, and P. Castillo-Borelley, "A CMOS integrated surface micromachined angular rate sensor: It's automotive applications," in Proc. Tech. Dig. 9th Int. Conf. on Solid-State Sensors and Actuators (Transducers '97), Chicago, IL, June 1997, pp. 851-854.

[12] I. Hopkin, "Performance and design of a silicon micromachined gyro," in Proc. Symp. Gyro Technology, Stuttgart, Germany, 1997, pp. 1.0-1.10

[13] A. J. Harris et al., "Issues associated with the design, fabrication and testing of a crystalline silicon ring gyroscope with electromagnetic actuation and sensing," J. Micromech. Microeng., pp. 284-292, 1998.

[14] F. Ayazi, "A high aspect-ratio high-performance polysilicon vibrating ring gyroscope," Ph.D. dissertation, Univ. Michigan, Jan. 2000.

[15] T. B. Gabrielson, "Mechanical-thermal noise in micromachined acoustic and vibration sensors," IEEE Trans. Electron Devices, vol. ED-40, pp. 903-909, May 1993.

[16] C. T. C. Nguyen, "Mechanical signal processors," Ph.D. dissertation, Univ. California at Berkeley, 1994.

[17] H. A. Rothbart, Mechanical Design Handbook. New York: McGrawHill, 1996.

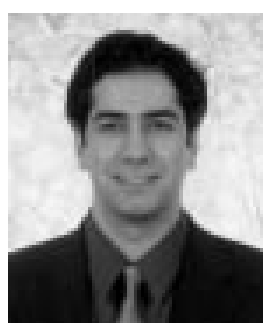

Farrokh Ayazi (S'95-M'00) was born in 1972. He received the B.S. degree in electrical engineering from the University of Tehran, Iran, in 1994 and the M.S. and Ph.D. degrees in electrical engineering from the University of Michigan, Ann Arbor, in 1997 and 2000, respectively.

In 2000, he joined the Faculty of Georgia Institute of Technology, Atlanta, where he is currently an Assistant Professor in the School of Electrical and Computer Engineering. His research interests are in the areas of MEMS, high aspect-ratio silicon micromachining technologies, analog integrated circuits, MEMS inertial sensors, RF MEMS, and integrated microsystems.

Dr. Ayazi is a member of Sigma Xi. He received a Rackham Predoctoral Fellowship from the University of Michigan for 1998-1999.

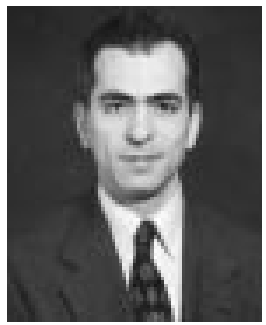

Khalil Najafi (S'84-M'86-SM'97-F'00) was born in 1958. He received the B.S., M.S., and the Ph.D. degree in 1980, 1981, and 1986, respectively, all in electrical engineering from the Department of Electrical Engineering and Computer Science, University of Michigan, Ann Arbor.

From 1986 to 1988 , he was employed as a Research Fellow, from 1988 to 1990, as an Assistant Research Scientist, from 1990 to 1993, as an Assistant Professor, from 1993 to 1998, as an Associate Professor, and since September 1998, as a Professor and the Director of the Solid-State Electronics Laboratory, Department of Electrical Engineering and Computer Science, University of Michigan. His research interests include: micromachining technologies, solid-state micromachined sensors, actuators, and MEMS; analog integrated circuits; implantable biomedical microsystems; hermetic micropackaging; and low-power wireless sensing/actuating systems.

Dr. Najafi was awarded a National Science Foundation Young Investigator Award from 1992 to 1997, was the recipient of the Beatrice Winner Award for Editorial Excellence at the 1986 International Solid-State Circuits Conference, of the Paul Rappaport Award for coauthoring the Best Paper published in the IEEE Transactions on Electron Devices, and of the Best Paper Award at ISSCC 1999. In 1994, he received the University of Michigan's "Henry Russel Award" for outstanding achievement and scholarship, and was selected as the "Professor of the Year" in 1993. In 1998, he was named the Arthur F. Thurnau Professor for outstanding contributions to teaching and research, and received the College of Engineering's Research Excellence Award. He has been active in the field of solid-state sensors and actuators for more than eighteen years, and has been involved in several conferences and workshops dealing with solid-state sensors and actuators, including the International Conference on Solid-State Sensors and Actuators, the Hilton-Head Solid-State Sensors and Actuators Workshop, and the IEEE/ASME Micro Electromechanical Systems (MEMS) Workshop. He is the Editor of Solid-State Sensors for IEEE Transactions on Electron Devices, Associate Editor for IEEE Transactions on Biomedical EngineERING, Associate Editor for IEEE JOURNAL OF SOLID-STATE CIRCUITS, and an Associate Editor for the JOURNAL OF Micromechanics AND MichroengineERING, Institute of Physics Publishing. 